\title{
PREDICTIVE DESIGN OF REVERSE INJECTION MECHANISM FOR ELECTROKINETIC DNA SAMPLE INJECTION
}

\author{
Manish Deshpande, Ken B. Greiner, John West and John R. Gilbert \\ Microcosm Technologies \\ 215 First Street, Cambridge MA 02142, USA \\ Luc Bousse and Abdel Minalla \\ Caliper Technologies Corp. \\ 605 Fairchild Drive, Mountain View, CA 94043
}

\begin{abstract}
A novel injector designed by CAD analyses is presented in this paper. The injection mechanism uses a pull-back inserted between the pinch and the switch stages of the typical cross injector to create a band that is more symmetric and narrower than the typical injector. The resulting separation efficiency is greatly enhanced as a result of the more optimal band shape. The injector was designed using a validated CAD tool, and verified subsequently by experiments. The resulting separation efficiency achieved was significantly greater than current injectors.
\end{abstract}

\section{INTRODUCTION}

Electrokinetic microfluidic microsystems are powerful analytical tools for many applications, such as nucleic acid analysis, enzyme assays, and immunoassays [1-4]. Such systems have gained considerable importance as components in micronscale integrated chemical/biochemical analysis or synthesis systems, also referred to as lab-on-a-chip. The basic "unit process" operations in these systems are sample injection, mixing, chemical reaction or modification, separation, and detection. Assembling a system of many "unit process" nodes requires one or more transport mechanisms to move sample and reagents through the "wires" of the system. Many of these systems rely on electrokinetic physics as their transport mechanism, although pressure and pneumatic applications have also been demonstrated. Complicated relationships exist between the microchannel geometries, the conditions under which the devices operate, and the behavior of the multicomponent fluids transported in these channels. In the past researchers have been forced to use costly trial and error methods to understand and design such microfluidic systems.

CAD tools can be a valuable aid in the design of microfluidic systems. Numerical analyses provide significant insight into the fluid mechanics in these systems. They allow the extraction of material and flow properties that are generally not well documented, or that vary from application to application or from one manufacturing technology to another. Furthermore such tools help the designer to explore a much larger space of designs than is easily available from experiment, and do so in a quantitative way that enables the extraction of key parameters for improved or optimal operation of common microchemical system components.
Simulations of electrokinetic flows have been reported in the literature for both electrophoretic $[5,6]$ and electroosmotic $[7,8]$ flows. In [6] and [8], simulations of pinched injection have been reported in channel intersections, demonstrating the application of electrical fields to position the species plug in the intersection, prior to switching. These analyses are $2 \mathrm{D}$ steady-state analyses with fixed field boundary conditions. Three-dimensional simulations of pinched injection have also been reported by these authors in previous work [9]. In all these cases, the analyses were aimed at demonstrating numerical capabilities and an understanding of the fundamental physics prevalent in the device. Agreement with experiment reported in these analyses is generally good.

The capabilities of CAD tools can only be fully realized when they are applied in predictive design of a device that improves or optimizes its performance. In this paper, we present the predictive use of simulation to design an improved injector, followed by experimental confirmation of that design and the use of the improved injector to achieve the fastest separations of DNA oligos currently achievable.

Microfluidic networks have a distinct advantage over conventional electrophoresis system in the area of rapid separations. Microfluidic systems have demonstrated separations in the order of seconds in comparison to several minutes in conventional systems $[3,4]$. A critical parameter in achieving rapid separations is the ability to inject a narrow plug into the separation column. Typically, microfluidic system achieve this by using pinched injection in a cross injection, as shown in Figure 2. The drive potential is supplemented

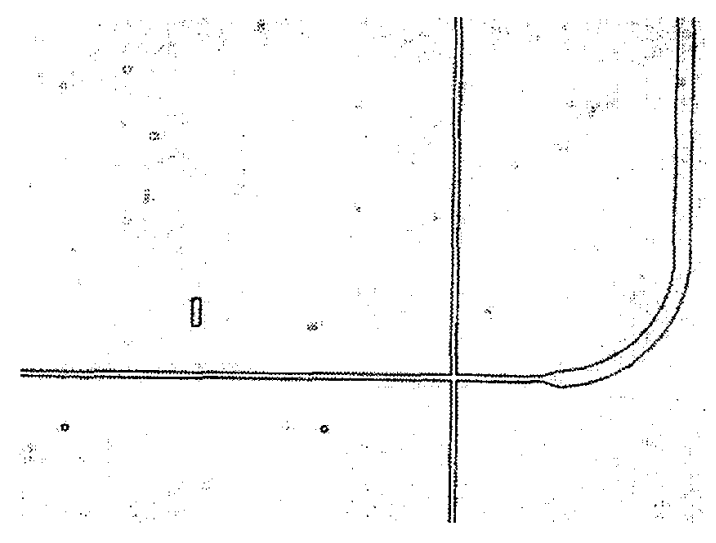

Figure 1. Photograph of a NS95 microchip used for the reverse injection experiments. 

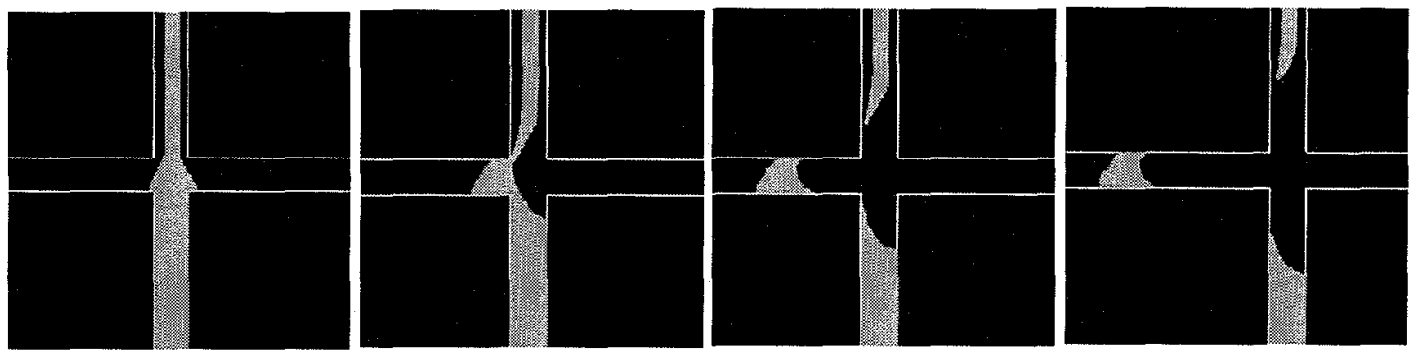

Figure 2. Typical Electrokinetic injection process showing the pinching and the switching process.

by fields in the transverse channels to shape the plug in the intersection prior to switching into the separation column as seen in the figure. The plug thus injected can be of the order of the channel dimension or smaller. As shown in Figure 2 the plug is broadencd in a trapezoidal shape. This broadening limits the possible resolution of the following separation column, causing the separation column to be longer than necessary. Designing injection to achieve a plug that is symmetric and narrower than the channel geometries is highly desirable.

Here, we will use CAD to focus on this aspect of the injection in an attempt to improve it. We will begin with a short description of the numerical tools followed by details of the simulation. Following that we will present experimental results that corroborate our findings, resulting in an improved injector, that demonstrates faster separations at lower voltages than conventional injectors.

\section{NUMERICAL FORMULATION}

The basic equations describing the fluid motion are the Navier-Stokes equations with appropriate electromigratory flux terms to represent the effect of the applied electric field on the charged species. The basis for electrophoresis is the differential migration of the charged species ions relative to the carrier molecules under the application of the external field. The differential migration is primarily an effect of the difference in the net charge between the solvent and solute ions, although frictional effects may also have some relevance. The migration velocity of the charged species can be expressed in terms of the applied field strength as $\mathrm{V}_{\mathrm{ep}}=$ $\mu_{\mathrm{ep}} \mathrm{E}$, where $\mu_{\mathrm{ep}}$ is the electrophoretic mobility of the ion in the carrier species. It is important to note that in most cases the carrier does not move under electrophoresis.

The motion of a charged species in the electric field can be determined by incorporating an electrokinetic transport mechanism in the species equation. The transport of the species is through the combined effect of the electroosmotic motion of the carrier fluid and the electrophoretic transport of the species under the effect of the applied electric field.

The numerical analyses presented above are derived under the following assumptions -

- Neutral Carrier: The carrier fluid is assumed to be electroneutral everywhere, except within the double layer.

- Dilute Sample: The carrier fluid is assumed to be predominant in calculating the physical properties of the fluid.
- Uncoupled Transport: Individual sample species do not affect each other as to their diffusion or mobilities.

- No Chemical Reactions: The charged sample species are assumed to be fully ionized in the mixture, and do not react with each other.

The above assumptions allow the density of the mixture to be assumed constant, reducing the problem to the incompressible form. The momentum and species equations are decoupled and can be solved separately.

The modeling of electrokinetic effects is incorporated into the FlumeCAD system. FlumeCAD is an integrated design environment consisting of 3D design, modeling and simulation software tools, which enable the creation and analysis of complex microfluidic devices. Inherent in the design flow implemented in FlumeCAD is the ability to translate from a layout and process view of the device to a solid model and to continue to a 3D device model allowing simulations that characterize the various physical phenomena present in the device. The numerical solution uses a three-dimensional finite element based engine as the back-end solver for the analyses.

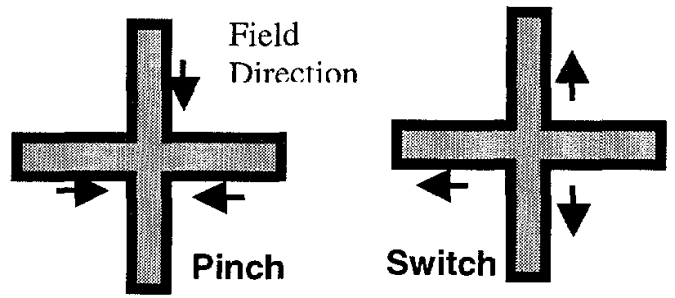

Figure 3. Schematic of the typical two phase injection

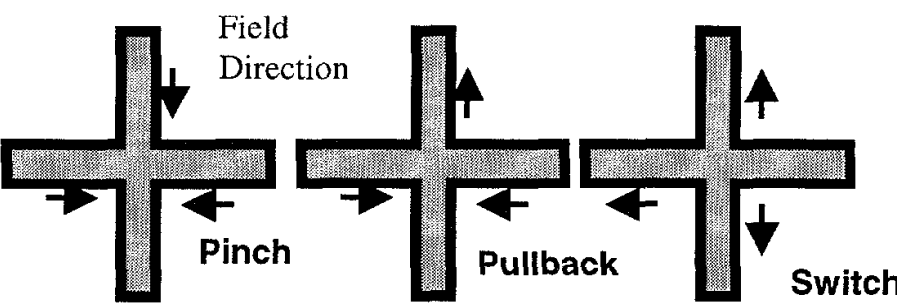

Figure 4. Schematic of Reverse Injection including pullback. 

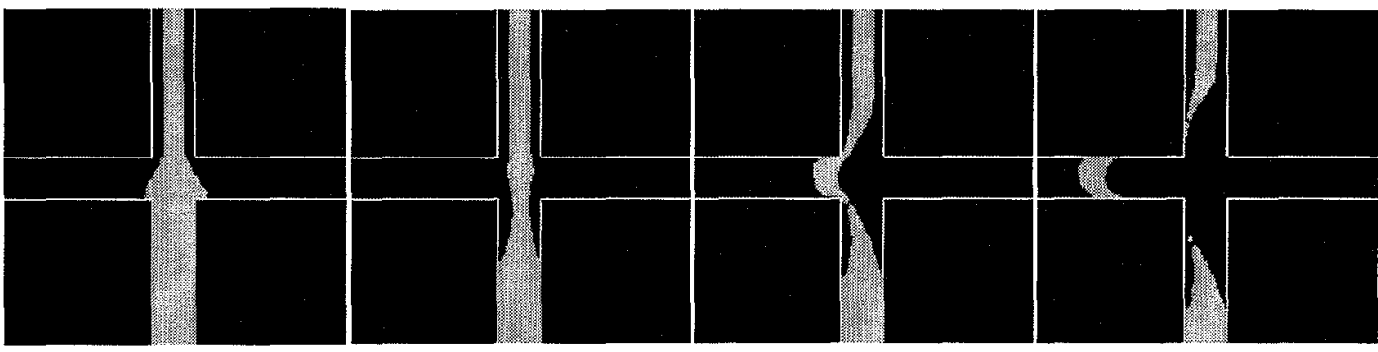

Figure 4. Reverse Injection process showing pull-back and subsequent switching into separation column.
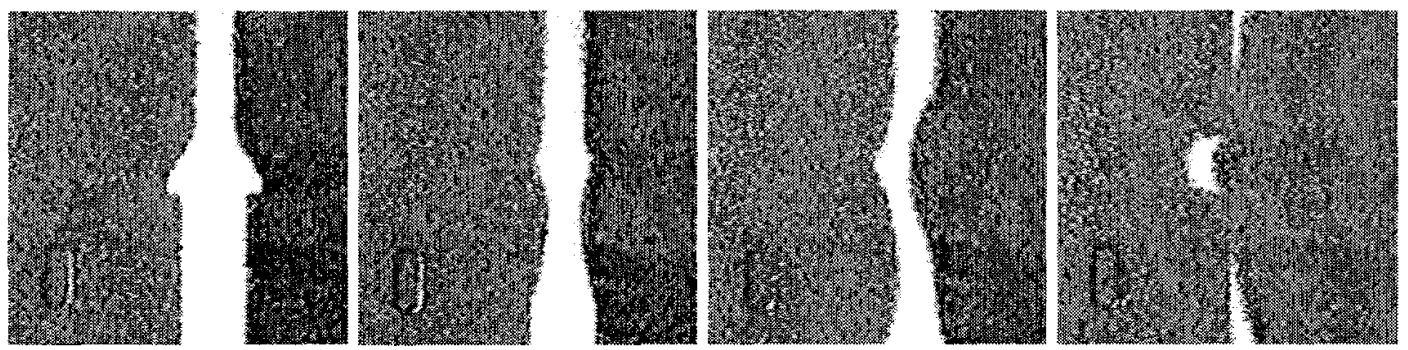

Figure 5. Experimental verification of reverse injection predicted by numerical simulations above.

\section{DESIGN ANAL YSIS}

The design analyses werc conducted on a cross injector. The numerical tools were first validated by comparing against experiment for the classic two-phase pinched injection. A schematic of this injection mechanism is shown in Figure 3 corresponding simulation results are shown in Figure 2. The injection consists of a "pinch" - fields in the transverse channels supplementing the drive current - followed by a "switch" sweeping the sample into the separation column along with current towards both top and bottom to separate the sample plug and prevent leakage. The simulations presented here were compared against corresponding experimental observations in [9] and show good qualitative agreement, indicating that the simulation tools capture all the relevant physics in the problem quite well.

As Figure 2 shows the switched plug in the separation column is trapezoidal in shape. The shape in the separation column is a consequence of the shape of the pinch in the intersection between the two channels. Since the sample is positioned in the intersection by a pinch, the shape in the intersection is trapezoidal, with the base of the trapezoid being wider than the characteristic dimension of the intersection. As the trapezoidal bands move downstream the individual fragments separate out based on their electrophoretic mobilities, and broaden based on their diffusivities. The effective band width in the separation column is determined by the width of the trapezoid base, which limits the separation efficiency.

A better injection mechanism is required to create a better sample plug in the intersection prior to separation. This can be achieved by alterations in the geometry or in the switching field sequence. One such example, showing a six-port switch, where the pinch and switch are carried out in different intersections, was presented in [10] and results in a narrower band in the separation column. A second approach termed "reverse injection", presented here, uses a three-phase switching sequence. The schematic of this sequence is shown in Figure 4. A "pull-back" phase is added between the pinch and the switch. In the creating of the pinch, the sample is positioned in the intersection by the pinching fields. Downstream of the intersection the band is significantly narrower than the width of the intersection, and is dependent on the strength of the pinching field. Adding a pull-back phase momentarily reverses the field in the drive channcl, whilc maintaining the pinching ficlds as scen in the schematic. The pull-back causes the downstream band to retract into the separation column creating a significantly narrower band at the entrance of the separation column. Additionally the downstream band is also symmetric since it is not modified by the effect of the pinch. The usual switching step then follows to drive the sample into the separation column.

Simulations showing the effect of the "pull-back" are presented in Figure 5. The effect on the band width and shape in the separation column is dramatic - the band is now both narrower and straighter than the conventional injector case in Figure 2. The results indicate that we can achieve DNA bands that are symmetric and narrower than the underlying channel geometry - consequently the resulting separation efficiency is expected to improve significantly as a result of the band shape.

\section{EXPERIMENTAL VERIFICATION}

Following the CAD analysis, experiments were conducted to verify the predictions presented in the previous section. The separations were carried out in soda-lime glass chips with channels $10 \mu \mathrm{m}$ deep and $30 \mu \mathrm{m}$ wide, filled with a $6 \%$ polyacrylamide-based separation matrix. The buffer was $100 \mathrm{mM}$ TAPS-Tris. For imaging 


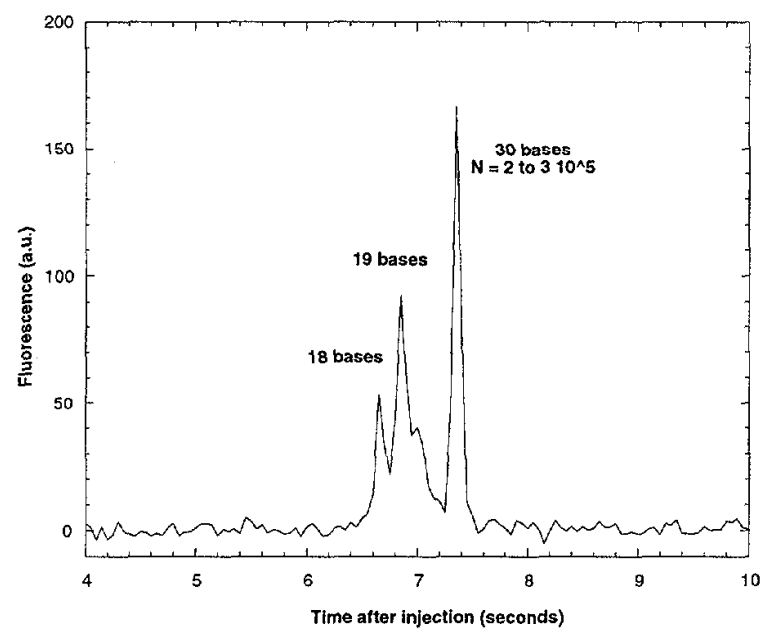

Figure 6. Ultra-high speed separations using reverse injection process.

studies, the samples were single-stranded DNA oligonucleotides at a concentration of $20 \mu \mathrm{M}$, fluorescently labeled at fluorescein wavelengths. Images were recorded on a Hammamatsu intensified CCD camera. For separations, similar oligonucleotides were used, but labeled at red wavelengths with Cy-5 dye, and at a concentration of $100 \mathrm{nM}$. Detection was carried out using a red diode laser, on a fluorescent microscope system as described earlier [3]. Experimental images corresponding to the reverse injection case are shown in Figure 6. The experimental results agree very well with the numerical predictions from Figure 5. The details of the sample behaviour including the shape in the intersection prior to switching, and the shape of the subsequently injected band, are correctly captured by the simulations. Separations from this injector are presented in Figure 6 . The narrow band created by reverse injection enabled the demonstration of 7 second DNA separations in a $0.5 \mathrm{~cm}$ long on-chip column, using only $150 \mathrm{~V}(300 \mathrm{~V} / \mathrm{cm})$. These are the fastest separations accomplished to date and are a result of the improved injector design emerging out of the CAD analyses.

\section{CONCLUSIONS}

The application of CAD in the design of microfluidic chip components is demonstrated in this paper, using the cross injector. In typical separations, the efficiency is limited by the width of the band in the separation column, which in turn is limited by the width of the band positioned in the intersection. Using a validated CAD tool, we have improved the injection characteristics by inserting a pull-back between the pinch and the switch. This injection, called "reverse injection" retracts the narrower and symmetric band downstream of the intersection back into the intersection, and subsequently into the separation column. The injection characteristics have been verified by experiment and have yielded faster separations using lower fields than have been accomplished hitherto.

The primary conclusion of this work is the successful application of CAD to predict and improve the performance of microtluidic components. CAD also has the advantage of being able to explore a much wider parameter space to design these improved components, and hence should serve as an important component of the design process.

\section{ACKNOWLEDGEMENTS}

This work was funded by DARPA Composite CAD (Grants no. F30602-98-2-0151 and F30602-96-2-0306).

\section{REFERENCES}

[1] D.J. Harrison, K. Fluri, K Seiler, Z. Fan, C.S. Effenhauser and A. Manz, "Micromachining a miniaturized capillary electrophoresis-based chemical analysis system on a chip," Science, 261 (1993) 895-897.

[2] S.C. Jacobson, R. Hergenroder, L.B. Koutny, R.J. Warmack and J.M. Ramsey, "Effects of Injection Schemes and Column Geometry on the Performance of Microchip Electrophoresis Devices," Anal. Chem., 66(1994), 1107.

[3] Luc Bousse, Bob Dubrow and Kathi Ulfelder, "High Performance DNA Separations in Microchip Electrophoresis Systems," u-TAS '98, 271.

[4] A.T. Woolley and R.A. Mathies, "Ultra High Speed DNA Sequencing using capillary electrophoresis chips," Anal. Chem., 65 (1995), 3676.

[5] X. C. Qui, L. Hu, J. H. Masliyah, and D. J. Harrison, "Understanding fluid mechanics within electrokinetically pumped microfluidic chips", 1997 International Conference on Solid-State Sensors and Actuators, Chicago, IL (1997).

[6] P.M. St. John, et al, "Metrology and Simulation of Chemical Transport in Microchannels", Solid State Sensors and Actuators Workshop, Hilton Head, SC (1998).

[7] N. A. Patankar and H. H. Hu, "Numerical Simulation of Electroosmotic Flow", Anal. Chem., 70, (1998).1870-81.

[8] Sergey V. Ermakov, S.C. Jacobson, and J.M. Ramsey, "Computer Simulations for Microchip Electrophoresis," uTAS '98, 149.

[9] Bousse, L., Minalla, A., Deshpande, M., Greiner, K., and Gilbert, J., "Optimization of Sample Injection Components in Electrokinetic Microfluidic Systems," MEMS '99, Orlando, FL (1999).

[10] Deshpande, M., Greiner, K., West, J., Gilbert, J., Bousse, L., and Minalla, A., "Novel Designs for Electrokinetic Injection in

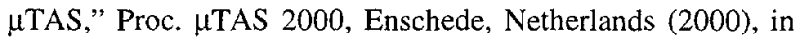
print.

[11] Bousse, L., "Electrokinetic Microfluidic Systems," SPIE Microfluidic Devices and Systems II, Santa Clara, CA (1999). 